\title{
Helifix Coil Device
}

National Cancer Institute

\section{Source}

National Cancer Institute. Helifix Coil Device. NCI Thesaurus. Code C49880.

A coil designed to allow a Helifix pacing electrode to be placed in the endocardium. 